\title{
Resistance Against Basil Downy Mildew in Ocimum Species
}

\author{
Yariv Ben-Naim, Lidan Falach, and Yigal Cohen
}

Faculty of Life Sciences, Bar-Ilan University, Ramat-Gan 5290002, Israel.

Accepted for publication 8 January 2015.

\begin{abstract}
Ben-Naim, Y., Falach, L., and Cohen, Y. 2015. Resistance against basil downy mildew in Ocimum species. Phytopathology 105:778-785.

Downy mildew, caused by the oomycete Peronospora belbahrii, is a devastating disease of sweet basil. In this study, 113 accessions of Ocimum species (83 Plant Introduction entries and 30 commercial entries) were tested for resistance against downy mildew at the seedling stage in growth chambers, and during three seasons, in the field. Most entries belonging to $O$. basilicum were highly susceptible whereas most entries belonging to $O$. americanum, $O$. kilimanadascharicum, O. gratissimum, O. campechianum, or $O$. tenuiflorum
\end{abstract}

ABSTRACT

Common sweet basil (Ocimum basilicum) is an economically important spice, extensively used for culinary purposes and as a source of essential oil and oleoresin for manufacturing perfumes and food flavors (Simon et al. 1990). The chemical composition of basil is an important source of antioxidants (Koroch et al. 2010) and antimicrobial agents (Elgayyar et al. 2001; Hussain et al. 2008 ) with a potential use in food preservation (Suppakul et al. 2003).

Basil includes more than 50 species of herbs and shrubs (Paton et al. 1999; Simon et al. 1990). The taxonomy of O. basilicum is complicated by existence of numerous botanical varieties, cultivars, and chemotypes that do not differ significantly in morphology (Simon et al. 1990). Interspecific hybridization and polyploidy occurs commonly within this genus (Harley and Heywood 1992) and creates taxonomic confusion in the understanding of the genetic relationships among the multitude number of Ocimum spp. (Paton and Putievsky 1996). Taxonomic issues in this genus based on morphology and chromosome number were recently reviewed based on molecular markers and nuclear DNA content (Aghaei et al. 2012; Carovic-Stanko et al. 2010; Vieira and Simon 2006). Basils also vary significantly in their morphology including growth habit, size, shape, texture, leaf orientation, and color of leaf, stem, and flower (Phippen and Simon 2000), resulting in a wide variety of ornamental cultivars (Simon et al. 1990).

The demand for fresh-market basil has grown in recent years due to increased consumer demand which has led to more intensive field and greenhouse production systems (Homa et al. 2014). Downy mildew caused by the oomycete Peronospora belbahrii is a limiting factor in basil production (Belbahri et al. 2005) and integrated management programs for basil diseases have been proposed (Garibaldi et al. 2007).

Downy mildew of basil was first described in Uganda in 1933 (Hansford 1933). In Israel, it was first reported in 2011 (Cohen et al. 2013b). The disease occurs in Europe (Garibaldi et al. 2005; Garibaldi et al. 2004a; Thines et al. 2009), South America (Ronco et al. 2009), the United States (Roberts et al. 2009), and Africa

Corresponding author: Y. Cohen; E-mail address: yigal.cohen1@gmail.com were highly resistant at both the seedling stage and the field. Twenty-seven highly resistant individual plants were each crossed with the susceptible sweet basil 'Peri', and the F1 progeny plants were examined for disease resistance. The F1 plants of two crosses were highly resistant, F1 plants of 24 crosses were moderately resistant, and F1 plants of one cross were susceptible, suggesting full, partial, or no dominance of the resistance gene(s), respectively. These data confirm the feasibility of producing downy mildew-resistant cultivars of sweet basil by crossing with wild Ocimum species.

Additional keywords: herbs, hybrids, spices, wild basils.

(McLeod et al. 2006). No reports are available on the occurrence races or pathotypes of $P$. belbahrii, except for isolates resistant to the fungicide mefenoxam (Cohen et al. 2013b).

Basil downy mildew (BDM) develops during periods of moderate temperatures, high air humidity, and prolonged leaf wetness (Garibaldi et al. 2005,2007). Frequently, infection is found immediately after seeding, suggesting that the pathogen can also be spread through contaminated seed (Farahani-Kofoet et al. 2012; Garibaldi et al. 2004b). P. belbahrii is disseminated by airborne spores produced at night under moist conditions. Spores infect leaf lamina with a single germ tube that penetrates into the epidermal cell walls and produces intercellular mycelium with haustoria in the mesophyll. Chlorotic lesions appear in leaves at 6 to 10 days postinoculation (dpi), depending on temperature, with $25^{\circ} \mathrm{C}$ being optimal. Sporulation takes place within $7 \mathrm{~h}$ at $100 \%$ relative humidity, in darkness only, with 18 to $20^{\circ} \mathrm{C}$ being optimal (Cohen et al. 2013a). Basil plants showing symptoms are unmarketable, including those with latent symptoms that deteriorate in storage.

Only a few fungicides are registered for control of the disease in Europe (Gilardi et al. 2013) and the United States (Homa et al. 2014). In the United States, mono- and dipotassium salts of phosphorous acid provided the best control, whereas moderate disease suppression was provided by mandipropamid, cyazofamid, and fiuopicolide (Homa et al. 2014). In Israel, best control was achieved with mefenoxam, mandipropamid, dimethomorph, and azoxystrobin, while moderate control was seen with mono- or dipotassium salts of phosphorous acid (Y. Cohen, M. Vaknin, and Y. Ben-Naim, unpublished data). However, mefenoxam became ineffective due to the appearance of resistant isolates (Cohen et al. 2013b) and some isolates show reduced sensitivity to dimethomorph and mandipropamid (unpublished data).

Darkness is essential for $P$. belbahrii to sporulate on infected basil leaves. When exposed to light during the sporulation period the pathogen produces sporophores but fails to produce spores. Indeed, nocturnal illumination in net-houses, which suppress spore production, was recently reported to effectively control the disease (Cohen et al. 2013a).

One of the most effective measures for disease control is via genetic manipulation of the host, namely introgression of resistant genes. The objectives of this study were to expand on what others 
have reported on potential sources of resistance to BDM in some species of Ocimum (Farahani-Kofoet et al. 2014; Wyenandt et al. 2010) and explore additional sources of resistance genes against BDM among commercial and wild basil species. Pyne et al. (2014) developed a rapid screening approach to identify resistance to $\mathrm{BDM}$ at the cotyledon growth stage. Field evaluations of basils under high downy mildew pressure confirmed the applicability of their screening approach to identify resistance to basil downy mildew. We performed parallel screening for resistance in the field and growth chambers and found them highly correlative.

\section{MATERIALS AND METHODS}

Germplasm. Eighty wild and cultivated basil species (Ocimum spp.) were obtained from the U.S. Department of AgricultureAgriculture Research Service Plant Introduction Station, Iowa State University, Ames, Iowa, and 33 sweet basil cultivars and ornamental basil lines were obtained from Richter's Herbs (Goodwood, Ontario, Canada), Hishtil (Petach Tikva, Israel), Flower Goddess (Beijing, China), Georgia vines, (Claxston, GA), and Frankonia Samen (Miltenberg, Germany). The origin of each entry is listed in Table 1. Leaf morphology as shown in Table 1 was assessed according to Wyenandt et al. (2010).

Plants. Seeds were sown in multicell trays (cell size $2.5 \mathrm{~cm}$ ) filled with a potting mixture (peat: vermiculite, $1: 1$, vol/vol), two to three seeds per cell, and grown in the greenhouse. At the four-leaf stage, one set of plants was inoculated and another set was planted in the field in a net house covered with a 50 mesh insect-proof net. This procedure was repeated (in replicated trials) three times in 2014, in 2 to 3 months intervals.

Pathogen. Twenty-one isolates of $P$. belbahrii were collected from the major growing regions in Israel during December 2011 to October 2014. The isolates collected until late 2012 were sensitive to mefenoxam while those collected during 2013 to 2014 were mostly resistant to this fungicide (Cohen et al. 2013b). The mefenoxamresistant isolate Knafo (collected in 2013 at Ein-Tamar, Southern Jordan Valley, Israel) was used in the experiments described below, unless stated otherwise. The isolates were maintained by repeated inoculation of sweet basil 'Peri' (Volcani Institute, Israel) at $20^{\circ} \mathrm{C}$.

Inoculation and disease assessment in seedlings. To inoculate the four-leaf stage plants, fresh spores of $P$. belbahrii were collected from sporulating plants into cold distilled water, adjusted to 5,000 spores $/ \mathrm{ml}$, and sprayed onto the upper leaf surfaces of the test plants. Plants were placed in a dew chamber overnight at $18^{\circ} \mathrm{C}$ to ensure infection and thereafter at $25^{\circ} \mathrm{C}$ under continuous illumination $\left(60 \mu\right.$ mole $\left.\mathrm{m}^{2} \mathrm{~s}^{-1}\right)$ for 6 days to ensure pathogen colonization. Plants were returned to the dew chamber at 7 dpi to enable sporulation of the pathogen on the inoculated plants. Sporulation intensity was visually estimated using an ordered categorical 0-to-3 scale, similar to Wyenandt et al. (2010) and Homa et al. (2014), in which $0=$ no visible sporulation; $1=$ scarce sporulation; $2=$ moderate sporulation; and $3=$ heavy sporulation.

Inoculation and disease assessment in the field. At 7 to 10 days after planting, when plants reached the six- to eight-leaf stage, they were spray-inoculated with $P$. belbahrii. Inoculation $(5,000$ spores $/ \mathrm{ml})$ was done at about 8 P.M. to ensure high humidity during infection. Starting at 1 week after inoculation, disease records were taken from the inoculated plants. Each plant was visually estimated for (i) incidence rating, the proportion of infected foliage; (ii) severity rating, the proportion of infected leaf area; and (iii) sporulation intensity. The three estimates were compiled visually using an ordered categorical 0 -to- 4 scale in which $0=$ no visible symptoms; 0.1 to $0.9=$ lesions occupy 10 to $90 \%$ of a leaf area; up to $5 \%$ of the leaves in a plant show symptoms but no sporulation is seen; 1 to $1.9=$ about 6 to $25 \%$ of the leaves show symptoms, sporulation is scarce; 2 to $2.9=$ about 26 to $50 \%$ of the leaves show symptoms, sporulation is moderate; and 3 to $4=$ about 51 to $100 \%$ of the leaves show symptoms, sporulation is heavy (Fig. 1).

Analysis of variance was calculated using the software JMP (SAS version 8). Means were compared using Turkey's honest significant difference test with $\alpha=0.05$.

At the end of the first season, seeds were collected from a most resistant individual plant in each entry and used for the second field trial. During the second season, crosses were made between the most resistant individual plant in each entry and the susceptible sweet basil Peri. F1 plants (4 to 10 plants per entry) were grown in the field in the third season. The inoculation procedure performed in the first season was similarly repeated in the second and the third seasons.

\section{RESULTS}

Table 1 provides data on the 113 entries of Ocimum species that were grown in the field during April to May 2014, including leaf morphology, uniformity of plant morphology, and response to infection with $P$. belbahrii (BDM, basil downy mildew). The total number of plants tested per entry ranged between 7 and 20. Leaves showed various morphologies (Wyenandt et al. 2010): fold up (UP), fold down (D), flat (FL), flat-down (FL-D), or flat-up (FL-UP). Most entries were uniform in leaf shape and plant morphology except those indicated with a star. A clear difference in response to BDM was observed between the four groups of entries (A to D). All entries (except entry number 70$)$ in group A $(O$. americanum and $O$. kilimandscharicum) were highly resistant. In group B (O. tenuiflorum, O. gratissimum, and $O$. campechianum), 11 entries were highly resistant and three entries were moderately resistant. In group C (O. basilicum and Ocimum spp.), eight entries were highly resistant and eight entries were moderately resistant. All 65 entries of group $\mathrm{D}($ O. basilicum $)$ were susceptible. In this group, all entries were uniform in response to BDM except 13,37 , and 58, which were susceptible except a few plants that were resistant (indicated with a double star).

Figure 2A provides a graphic illustration of the above results. A clear distinction in disease intensity is seen between the four groups. Mean disease intensity for the entries in groups A, B, C, and D was $0.33,0.45,0.96$, and 3.73 , respectively. Disease intensity was recorded three times during the epidemic (27 days). Values of the final score are presented in Table 1 and illustrated in Figure 2A.

The mean values ( 7 to 40 plants per entry) of disease intensity in four-leaf plants at 7 days after artificial inoculation in growth

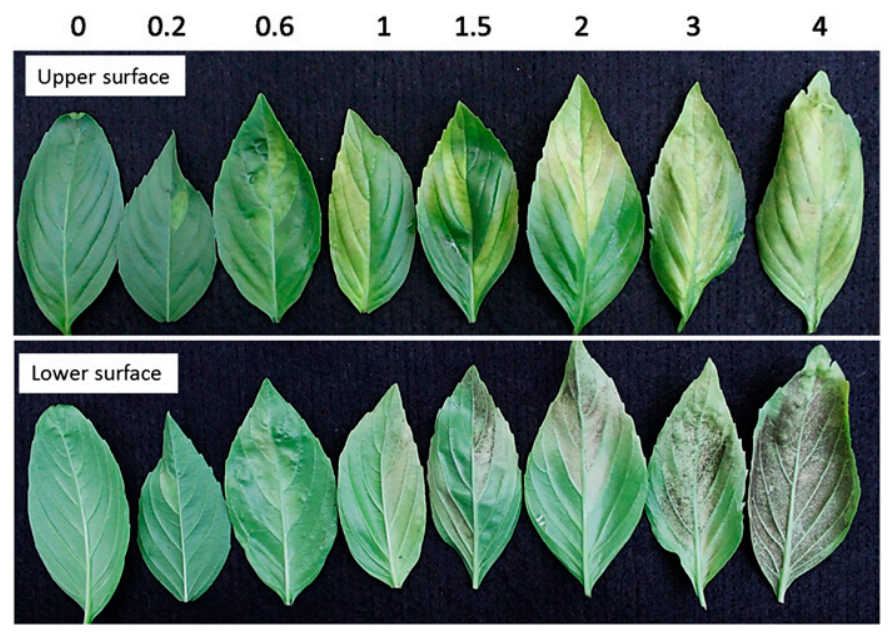

Fig. 1. Symptoms on upper leaf surfaces and sporulation on lower leaf surfaces of downy mildew in basil leaves caused Peronospora belbahrii. Figures on top indicate the level of disease intensity on a 0 to 4 visual scale. This scale was used to assess the susceptibility/resistance to downy mildew of 113 accessions of Ocimum species in the field. 
chambers are shown in Figure 2B. Several entries in groups A, B, and $\mathrm{C}$ were highly resistant to the disease, while most entries in group D were susceptible.

To verify that resistance holds against other isolates of the pathogen, 12 accessions were inoculated at the four-leaf stage with each of 11 isolates of $P$. belbahrii, four sensitive and seven resistant to mefenoxam, representing the various growing regions in the country. Accessions PI 500944, PI 500945, PI 500950, PI 500951,
PI 414201, and PI 652059 were highly resistant (disease intensity 0 to 0.5 ). PI 17297 and PI 172998 were resistant (disease intensity 0.5 to 0.9 ), Magic White and PI 652054 were moderately resistant (disease intensity 1.1 to 2.2), and the commercial cultivars Peri and Genovese were highly susceptible (disease intensity 3.5 to 4 ) to all isolates.

The disease intensity values obtained in seedlings in growth chambers stand in good correlation $(R=0.73$ for all 113 entries)

TABLE 1. Response to downy mildew of 113 entries of Ocimum accessions under field conditions

\begin{tabular}{|c|c|c|c|c|c|c|c|c|}
\hline \multirow[b]{2}{*}{ Entry } & \multirow[b]{2}{*}{$\mathrm{PI} /$ species/cultivar } & \multirow[b]{2}{*}{ Botanical name } & \multirow[b]{2}{*}{ Origin/supplier } & \multirow[b]{2}{*}{ Leaf shape ${ }^{x}$} & \multicolumn{3}{|c|}{ Number of plants ${ }^{\mathrm{y}}$} & \multirow[b]{2}{*}{ Score ${ }^{\mathrm{z}}$} \\
\hline & & & & & Total & $\mathrm{R}(\leq 0.9)$ & $\mathrm{S}(\geq 1)$ & \\
\hline \multicolumn{9}{|c|}{ Group A: $O$. americanum and $O$. kilimandscharicum } \\
\hline 3 & PI 500945 & O. americanum var. americanum & Zambia & $\mathrm{D}$ & 9 & 9 & 0 & $0.00 \mathrm{~d}$ \\
\hline 67 & PI 500942 & O. americanum var. pilosum & Zambia & FL-D & 9 & 9 & 0 & $0.00 \mathrm{~d}$ \\
\hline 69 & PI 500944 & O. americanum var. pilosum & Zambia & FL-UP* & 9 & 9 & 0 & $0.00 \mathrm{~d}$ \\
\hline 71 & PI 500948 & O. americanum var. pilosum & Zambia & FL & 9 & 9 & 0 & $0.00 \mathrm{~d}$ \\
\hline 73 & PI 500950 & O. americanum var. pilosum & Zambia & FL-UP* & 9 & 9 & 0 & $0.00 \mathrm{~d}$ \\
\hline 76 & PI 500954 & O. americanum var. pilosum & Zambia & UP & 9 & 9 & 0 & $0.00 \mathrm{~d}$ \\
\hline 77 & PI 652052 & O. kilimandscharicum & Zambia & $\mathrm{F}^{*}$ & 9 & 9 & 0 & $0.00 \mathrm{~d}$ \\
\hline 74 & PI 500951 & O. americanum var. pilosum & Zambia & $\mathrm{D}$ & 9 & 9 & 0 & $0.08 \mathrm{c}$ \\
\hline 2 & PI 254352 & O. americanum var. americanum & Iraq & FL & 9 & 9 & 0 & $0.10 \mathrm{c}$ \\
\hline 72 & PI 500949 & O. americanum var. pilosum & Zambia & FL-UP & 9 & 9 & 0 & $0.13 \mathrm{c}$ \\
\hline 54 & PI 652053 & O. americanum var. americanum & USA, Maryland & $\mathrm{FL}$ & 15 & 15 & 0 & $0.17 \mathrm{c}$ \\
\hline 75 & PI 500953 & O. americanum var. pilosum & Zambia & $\mathrm{D}$ & 9 & 7 & 2 & $0.38 \mathrm{bc}$ \\
\hline 1 & PI 253158 & O. americanum var. americanum & Iran & FL & 9 & 9 & 0 & $0.43 \mathrm{bc}$ \\
\hline 68 & PI 500943 & O. americanum var. pilosum & Zambia & FL-D & 9 & 7 & 2 & $0.46 \mathrm{bc}$ \\
\hline 6 & PI 652062 & O. americanum var. americanum & Tanzania & FL & 9 & 9 & 0 & $0.50 \mathrm{bc}$ \\
\hline 4 & PI 652058 & O. americanum var. americanum & Togo & FL & 9 & 6 & 3 & $0.65 \mathrm{bc}$ \\
\hline 5 & PI 652060 & O. americanum var. americanum & Pakistan & FL & 9 & 5 & 4 & $0.96 \mathrm{~b}$ \\
\hline 70 & PI 500947 & O. americanum var. pilosum & Zambia & FL & 9 & 0 & 9 & $2.03 \mathrm{a}$ \\
\hline \multicolumn{9}{|c|}{ Group B: O. tenuiflorum, O. gratissimum, and $O$. campechianum } \\
\hline 98 & O. tenuiflorum-1 & O. tenuiflorum & Georgia vines, USA & $\mathrm{D}$ & 20 & 20 & 0 & $0.00 \mathrm{e}$ \\
\hline 99 & O. gratissimum-1 & O. gratissimum & Georgia vines, USA & FL & 20 & 20 & 0 & $0.00 \mathrm{e}$ \\
\hline 78 & PI 652066 & O. campechianum & Brazil & FL & 9 & 9 & 0 & $0.00 \mathrm{e}$ \\
\hline 79 & PI 652067 & O. gratissimum & Brazil & FL & 9 & 9 & 0 & $0.00 \mathrm{e}$ \\
\hline 80 & PI 211715 & O. gratissimum var. gratissimum & Taiwan & FL & 9 & 9 & 0 & $0.00 \mathrm{e}$ \\
\hline 81 & PI 500952 & O. gratissimum var. gratissimum & Zambia & FL & 9 & 9 & 0 & $0.00 \mathrm{e}$ \\
\hline 82 & PI 652055 & O. gratissimum var. macrophyllum & Sri Lanka & FL & 9 & 9 & 0 & $0.00 \mathrm{e}$ \\
\hline 64 & PI 414205 & O. tenuiflorum & USA, Maryland & FL & 15 & 15 & 0 & $0.37 \mathrm{de}$ \\
\hline 61 & PI 414202 & O. tenuiflorum & USA, Maryland & FL & 15 & 13 & 2 & $0.51 \mathrm{de}$ \\
\hline 60 & PI 414201 & O. tenuiflorum & USA, Maryland & FL & 15 & 10 & 5 & $0.60 \mathrm{~cd}$ \\
\hline 66 & PI 652059 & O. tenuiflorum & Maldives & FL & 15 & 8 & 7 & $0.79 \mathrm{bcd}$ \\
\hline 62 & PI 414203 & O. tenuiflorum & USA, Maryland & FL & 15 & 6 & 9 & $1.09 \mathrm{bc}$ \\
\hline 65 & PI 652056 & O. tenuiflorum & Denmark & FL & 15 & 2 & 13 & $1.18 \mathrm{ab}$ \\
\hline 63 & PI 414204 & O. tenuiflorum & USA, Maryland & FL & 15 & 0 & 15 & $1.73 \mathrm{a}$ \\
\hline \multicolumn{9}{|c|}{ Group C: O. basilicum var. and Ocimum spp. } \\
\hline 107 & Spice basil & Ocimum spp. & Richter's, Canada & FL & 10 & 9 & 1 & $0.15 \mathrm{e}$ \\
\hline 22 & PI 211586 & O. basilicum var. thyrsiflorum & Afghanistan & FL, UP & 15 & 12 & 3 & $0.20 \mathrm{de}$ \\
\hline 113 & Blue Spice & Ocimum spp. & Richter's, Canada & FL & 7 & 7 & 0 & 0.30 bcde \\
\hline 12 & PI 172998 & O. basilicum var. anisatum & Turkey & FL & 15 & 15 & 0 & 0.38 cde \\
\hline 11 & PI 172997 & O. basilicum var. anisatum & Turkey & FL & 20 & 20 & 0 & 0.50 bcde \\
\hline 10 & PI 172996 & O. basilicum var. anisatum & Turkey & FL* & 20 & 20 & 0 & 0.57 bcde \\
\hline 94 & Magic Mountain F1 & O. kilimandscharicum $\times O$. basilicum & Hishtil, Israel & FL-D & 20 & 15 & 5 & 0.58 bcde \\
\hline 95 & Magic White F1 & O. kilimandscharicum $\times O$. basilicum & Hishtil, Israel & FL-D & 20 & 15 & 5 & 0.58 bcde \\
\hline 86 & Wild Color F1 & O. basilicum & Hishtil, Israel & FL & 20 & 0 & 20 & $1.30 \mathrm{abcd}$ \\
\hline 26 & PI 296391 & O. basilicum var. citrodorum & Iran & $\mathrm{FL}$ & 15 & 7 & 8 & $1.30 \mathrm{abcd}$ \\
\hline 8 & PI 170579 & O. basilicum var. minimum & Turkey & UP & 20 & 0 & 20 & $1.34 \mathrm{abc}$ \\
\hline 14 & PI 174284 & O. basilicum var. anisatum & Turkey & FL* & 12 & 7 & 5 & $1.38 \mathrm{ab}$ \\
\hline 55 & PI 652054 & O. basilicum var. citrodorum 'Mrs. Burns' & USA, New Mexico & FL-D & 15 & 0 & 15 & $1.47 \mathrm{ab}$ \\
\hline 23 & PI 253157 & O. basilicum var. citrodorum & Iran & FL-D & 16 & 6 & 10 & $1.73 \mathrm{a}$ \\
\hline 105 & Lemon basil & O. basilicum var. citrodorum & Richter's, Canada & FL-D & 9 & 1 & 9 & $1.79 \mathrm{a}$ \\
\hline 84 & Ajaka F1 & O. basilicum & Hishtil, Israel & UP & 20 & 0 & 20 & $1.81 \mathrm{a}$ \\
\hline \multicolumn{9}{|c|}{ Group D: O. basilicum } \\
\hline 102 & Sweet Danni & O. $\times$ Citrodorum & Richter's, Canada & FL-D & 12 & 0 & 12 & $2.15 \mathrm{c}$ \\
\hline 83 & PI 500946 & Ocimum spp. & Zambia & $\mathrm{F}^{*}$ & 9 & 0 & 9 & $2.28 \mathrm{c}$ \\
\hline 37 & PI 368697 & O. basilicum & Macedonia & $\mathrm{FL}^{* *}$ & 14 & 1 & 13 & $2.33 \mathrm{c}$ \\
\hline
\end{tabular}

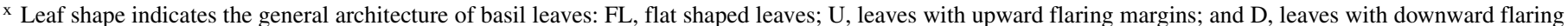
margins. * indicates lack of uniformity of plant morphology in the accession. ** indicates lack of uniformity of plant resistance in the accession.

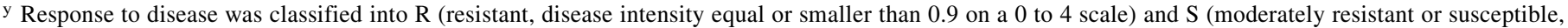
disease intensity equal or larger than 1.0).

z Scores (mean) followed by the same letter are not significantly different according to least significant difference test at $P \leq 0.05$. 
with the disease intensity values obtained in the field at 27 dpi. Twelve entries were resistant in growth chamber assays but responded susceptible in the field $(R=0.31)$ and six entries were susceptible in growth chamber assays while responding resistant in the field $(R=0.55)$. All other 95 entries were either resistant or susceptible in both the seedling stage and the field $(R=0.83)$.

At the end of the first season in the field (May 2014), seeds were collected from a single, most resistant plant of 40 entries (Table 2) and retested for BDM resistance under field conditions during June to July 2014 (second consecutive season). Seeds were collected from a single, most resistant plant at the end the second season in July 2014 and retested in a third consecutive season for BDM resistance under field conditions during August to September 2014. From group D, eight entries were selected for the third retesting trial: Sweet Danni, which was reported resistant (Wyenandt et al. 2010), three highly resistant individuals from the segregating entries 13,37 , and 58, and four highly susceptible ones as controls.

TABLE 1. (continued from prevoius page)

\begin{tabular}{|c|c|c|c|c|c|c|c|c|}
\hline \multirow[b]{2}{*}{ Entry } & \multirow[b]{2}{*}{$\mathrm{PI} /$ species/cultivar } & \multirow[b]{2}{*}{ Botanical name } & \multirow[b]{2}{*}{ Origin/supplier } & \multirow[b]{2}{*}{ Leaf shape ${ }^{\mathrm{x}}$} & \multicolumn{3}{|c|}{ Number of plants ${ }^{\mathrm{y}}$} & \multirow[b]{2}{*}{ Score $^{\mathrm{z}}$} \\
\hline & & & & & Total & $\mathrm{R}(\leq 0.9)$ & $\mathrm{S}(\geq 1)$ & \\
\hline 9 & PI 170581 & O. basilicum var. minimum & Turkey & UP* & 20 & 0 & 20 & $2.55 \mathrm{bc}$ \\
\hline 56 & PI 652061 & O. basilicum & India & FL & 14 & 0 & 14 & $2.67 \mathrm{bc}$ \\
\hline 20 & PI 197442 & O. basilicum & Ethiopia & FL & 15 & 0 & 15 & $2.83 \mathrm{bc}$ \\
\hline 13 & PI 173746 & O. basilicum & Turkey & FL-D** & 16 & 0 & 16 & $2.95 \mathrm{bc}$ \\
\hline 109 & Rubin & O. basilicum & Richter's, Canada & $\mathrm{D}$ & 10 & 0 & 10 & $3.15 \mathrm{~b}$ \\
\hline 104 & Bush basil & O. basilicum var. minimum & Richter's, Canada & UP & 10 & 0 & 10 & $3.20 \mathrm{~b}$ \\
\hline 110 & Globette & O. basilicum & Richter's, Canada & $\mathrm{F}$ & 10 & 0 & 10 & $3.20 \mathrm{~b}$ \\
\hline 106 & Queentte Thai & O. basilicum & Richter's, Canada & UP & 12 & 0 & 12 & $3.45 \mathrm{~b}$ \\
\hline 58 & PI 652070 & O. basilicum 'Sweet basil' & USA, Pennsylvania & $\mathrm{D} * *$ & 15 & 1 & 14 & $3.46 \mathrm{~b}$ \\
\hline 21 & PI 207498 & O. basilicum & Afghanistan & FL & 15 & 0 & 15 & $3.50 \mathrm{~b}$ \\
\hline 50 & PI 414199 & O. basilicum & USA, Maryland & D* & 16 & 0 & 16 & $3.50 \mathrm{~b}$ \\
\hline 108 & Spicy Globe & O. basilicum var. minimum & Richter's, Canada & UP & 14 & 0 & 14 & $3.51 \mathrm{~b}$ \\
\hline 103 & Mintte basil & O. basilicum var. minimum & Richter's, Canada & UP & 10 & 0 & 10 & $3.67 \mathrm{ab}$ \\
\hline 111 & Corsican basil & O. basilicum & Richter's, Canada & $\mathrm{D}$ & 15 & 0 & 15 & $3.71 \mathrm{ab}$ \\
\hline 112 & Gecofure basil & O. basilicum 'Genovese' & Richter's, Canada & $\mathrm{D}$ & 12 & 0 & 12 & $3.73 \mathrm{ab}$ \\
\hline 44 & PI 414193 & O. basilicum & USA, Maryland & UP* & 20 & 0 & 20 & $3.74 \mathrm{ab}$ \\
\hline 93 & Wild Magic F1 & O. basilicum & Hishtil, Israel & $\mathrm{D}$ & 20 & 0 & 20 & $3.75 \mathrm{ab}$ \\
\hline 91 & Dark Lady F1 & O. basilicum & Hishtil, Israel & $\mathrm{D}$ & 20 & 0 & 20 & $3.80 \mathrm{ab}$ \\
\hline 97 & Sweet basil & O. basilicum 'Sweet basil' & F. Goddess, China & $\mathrm{D}$ & 20 & 0 & 20 & $3.81 \mathrm{ab}$ \\
\hline 25 & PI 296390 & O. basilicum var. thyrsiflorum & Iran & FL & 16 & 0 & 16 & $3.83 \mathrm{ab}$ \\
\hline 88 & Habana F1 & O. basilicum & Hishtil, Israel & $\mathrm{D}$ & 20 & 0 & 20 & $3.88 \mathrm{ab}$ \\
\hline 89 & Red Ball F1 & O. basilicum & Hishtil, Israel & $\mathrm{D}$ & 20 & 0 & 20 & $3.88 \mathrm{ab}$ \\
\hline 36 & PI 368695 & O. basilicum & Macedonia & UP* & 18 & 0 & 18 & $3.89 \mathrm{ab}$ \\
\hline 42 & PI 379413 & O. basilicum & Macedonia & $\mathrm{D}$ & 20 & 0 & 20 & $3.90 \mathrm{a}$ \\
\hline 34 & PI 358471 & O. basilicum & Macedonia & UP & 18 & 0 & 18 & $3.94 \mathrm{a}$ \\
\hline 35 & PI 358472 & O. basilicum & Macedonia & $\mathrm{D}$ & 13 & 0 & 13 & $3.94 \mathrm{a}$ \\
\hline 100 & Peri & O. basilicum Sweet basil 'Peri' & Hishtil, Israel & $\mathrm{D}$ & 20 & 0 & 20 & $3.95 \mathrm{a}$ \\
\hline 17 & PI 176646 & O. basilicum & Turkey & UP & 18 & 0 & 18 & $3.95 \mathrm{a}$ \\
\hline 7 & PI 170578 & O. basilicum var. minimum & Turkey & UP & 20 & 0 & 20 & $4.00 \mathrm{a}$ \\
\hline 15 & PI 174285 & O. basilicum var. purpurascens & Turkey & $D^{*}$ & 15 & 0 & 15 & $4.00 \mathrm{a}$ \\
\hline 16 & PI 175793 & O. basilicum & Turkey & FL & 20 & 0 & 20 & $4.00 \mathrm{a}$ \\
\hline 18 & PI 182246 & O. basilicum var. purpurascens & Turkey & $D^{*}$ & 14 & 0 & 14 & $4.00 \mathrm{a}$ \\
\hline 19 & PI 190100 & O. basilicum & Iran & UP & 15 & 0 & 15 & $4.00 \mathrm{a}$ \\
\hline 24 & PI 263870 & O. basilicum & Greece & FL & 15 & 0 & 15 & $4.00 \mathrm{a}$ \\
\hline 27 & PI 358463 & O. basilicum & Macedonia & FL & 16 & 0 & 16 & $4.00 \mathrm{a}$ \\
\hline 28 & PI 358464 & O. basilicum var. thyrsiflorum & Serbia & FL-UP & 15 & 0 & 15 & $4.00 \mathrm{a}$ \\
\hline 29 & PI 358465 & O. basilicum & Macedonia & FL-UP & 12 & 0 & 12 & $4.00 \mathrm{a}$ \\
\hline 30 & PI 358466 & O. basilicum & Serbia & $\mathrm{D}$ & 15 & 0 & 15 & $4.00 \mathrm{a}$ \\
\hline 31 & PI 358467 & O. basilicum & Macedonia & UP & 15 & 0 & 15 & $4.00 \mathrm{a}$ \\
\hline 32 & PI 358468 & O. basilicum & Macedonia & UP & 16 & 0 & 16 & $4.00 \mathrm{a}$ \\
\hline 33 & PI 358469 & O. basilicum & Macedonia & $\mathrm{UP}^{*}$ & 18 & 0 & 18 & $4.00 \mathrm{a}$ \\
\hline 38 & PI 368698 & O. basilicum & Macedonia & FL-D & 15 & 0 & 15 & $4.00 \mathrm{a}$ \\
\hline 39 & PI 368699 & O. basilicum & Macedonia & FL-D & 15 & 0 & 15 & $4.00 \mathrm{a}$ \\
\hline 40 & PI 368700 & O. basilicum & Macedonia & UP* & 13 & 0 & 13 & $4.00 \mathrm{a}$ \\
\hline 41 & PI 379412 & O. basilicum & Macedonia & $\mathrm{D}$ & 15 & 0 & 15 & $4.00 \mathrm{a}$ \\
\hline 43 & PI 379414 & O. basilicum & Macedonia & $\mathrm{D}$ & 20 & 0 & 20 & $4.00 \mathrm{a}$ \\
\hline 45 & PI 414194 & O. basilicum & USA, Maryland & $\mathrm{FL}^{*}$ & 20 & 0 & 20 & $4.00 \mathrm{a}$ \\
\hline 46 & PI 414195 & O. basilicum & USA, Maryland & UP & 15 & 0 & 15 & $4.00 \mathrm{a}$ \\
\hline 47 & PI 414196 & O. basilicum & USA, Maryland & UP & 15 & 0 & 15 & $4.00 \mathrm{a}$ \\
\hline 48 & PI 414197 & O. basilicum & USA, Maryland & $\mathrm{UP} / \mathrm{D} *$ & 13 & 0 & 13 & $4.00 \mathrm{a}$ \\
\hline 49 & PI 414198 & O. basilicum & USA, Maryland & UP* & 14 & 0 & 14 & $4.00 \mathrm{a}$ \\
\hline 51 & PI 414200 & O. basilicum & USA, Maryland & UP & 17 & 0 & 17 & $4.00 \mathrm{a}$ \\
\hline 52 & PI 531396 & O. basilicum & USA, Maryland & UP & 15 & 0 & 15 & $4.00 \mathrm{a}$ \\
\hline 53 & PI 601365 & O. basilicum 'Purple Ruffles' & USA, Maryland & $\mathrm{D}$ & 15 & 0 & 15 & $4.00 \mathrm{a}$ \\
\hline 57 & PI 652065 & O. basilicum 'Genovese' & Italy & $\mathrm{D}$ & 12 & 0 & 12 & $4.00 \mathrm{a}$ \\
\hline 59 & PI 652071 & O. basilicum var. purpurascens 'Dark Opal' & USA, California & $\mathrm{D}$ & 14 & 0 & 14 & $4.00 \mathrm{a}$ \\
\hline 85 & Feronia & O. basilicum $\mathrm{F} 1$ & Hishtil, Israel & $\mathrm{D}$ & 20 & 0 & 20 & $4.00 \mathrm{a}$ \\
\hline 87 & Lhasa & O. basilicum F1 & Hishtil, Israel & $\mathrm{D}$ & 20 & 0 & 20 & $4.00 \mathrm{a}$ \\
\hline 90 & Green Ball & O. basilicum $\mathrm{F} 1$ & Hishtil, Israel & $\mathrm{D}$ & 20 & 0 & 20 & $4.00 \mathrm{a}$ \\
\hline 92 & Green Super Globe & O. basilicum F1 & Hishtil, Israel & $\mathrm{D}$ & 20 & 0 & 20 & $4.00 \mathrm{a}$ \\
\hline 96 & Pesto Perpetuo & O. basilicum $\mathrm{F} 1$ & Hishtil, Israel & $\mathrm{D}$ & 20 & 0 & 20 & $4.00 \mathrm{a}$ \\
\hline 101 & Genoveser 142 & O. basilicum 'Genovese' & Frankonia, Germany & $\mathrm{D}$ & 12 & 0 & 12 & $4.00 \mathrm{a}$ \\
\hline
\end{tabular}


During the second season crosses were made between the highly resistant individuals and the susceptible cultivar Peri. The F1 progeny plants were tested for resistance to BDM during the third season.

The results are given in Table 2. They confirm the high resistance of several entries in all three seasons. A correlation of 0.85 was calculated between the resistance values obtained in the first and the second seasons, and 0.89 between the second and the third seasons. In group $\mathrm{D}$, three entries segregated for resistance in the first season and the selected resistant individuals expressed high levels of resistance in the next two seasons.

Twenty-seven F1 hybrids were produced and tested for resistance. Their responses to BDM in the third season are given in Figure 3 along with the responses of their parents. Resistance of most parents was inherited as a partial dominant trait. Some parents, however, had a different mode of inheritance: the resistant entries 73 (PI 500950, O. americanum var. pilosum), 3 (PI 500945, O. americanum var. americanum), and 74 (PI 500951, O. americanum var. pilosum) probably carry a dominant gene(s) for resistance while entries 55 (O. basilicum var. citrodorum 'Mrs. Burns') and 60 (PI 414201, O. tenuiflorum) probably carry a dominant gene(s) for partial resistance. Entry 77 (PI 500954, O. kilimanadascharicum) was unique. Its resistance seemed to be inherited in a recessive manner (Fig. 3).

\section{DISCUSSION}

The recent outbreak of downy mildew in sweet basil in Israel (Cohen et al. 2013b) has caused enormous economical loses to growers due to the lack of adequate knowledge on the biology and control of the disease. This study was launched to provide relief to growers by developing basil lines resistant to the mildew. For this purpose we have looked for sources of resistance genes and evaluated 113 genotypes of basil (wild, cultivated, and ornamental) for resistance against $P$. belbahrii at the four-leaf stage in growth chambers and during three consecutive seasons in the field.

The 113 accessions were divided into four groups: group A, comprised of $O$. americanum and $O$. kilimandscharicum; group B, $O$. tenuiflorum, $O$. gratissimum, and $O$. campechianum; group C, O. basilicum and Ocimum spp.; and group D, O. basilicum. Mean disease intensity for the entries in groups A, B, C, and D at the fourleaf stage was $0.36,0.91,0.71$, and 2.30 ( 0 to 3 visual scale, data not shown) and in the field was $0.33,0.45,0.96$, and 3.73 ( 0 to 4 visual scale), respectively. This suggests that genes for immunity (disease intensity $=0$ ) or high resistance (disease intensity 0.1 to 0.9 ) reside mainly among wild species of Ocimum in groups A and B. Nevertheless, some entries of $O$. basilicum expressed high resistance (e.g., O. basilicum var. anisatum) or moderate resistance (e.g., O. basilicum

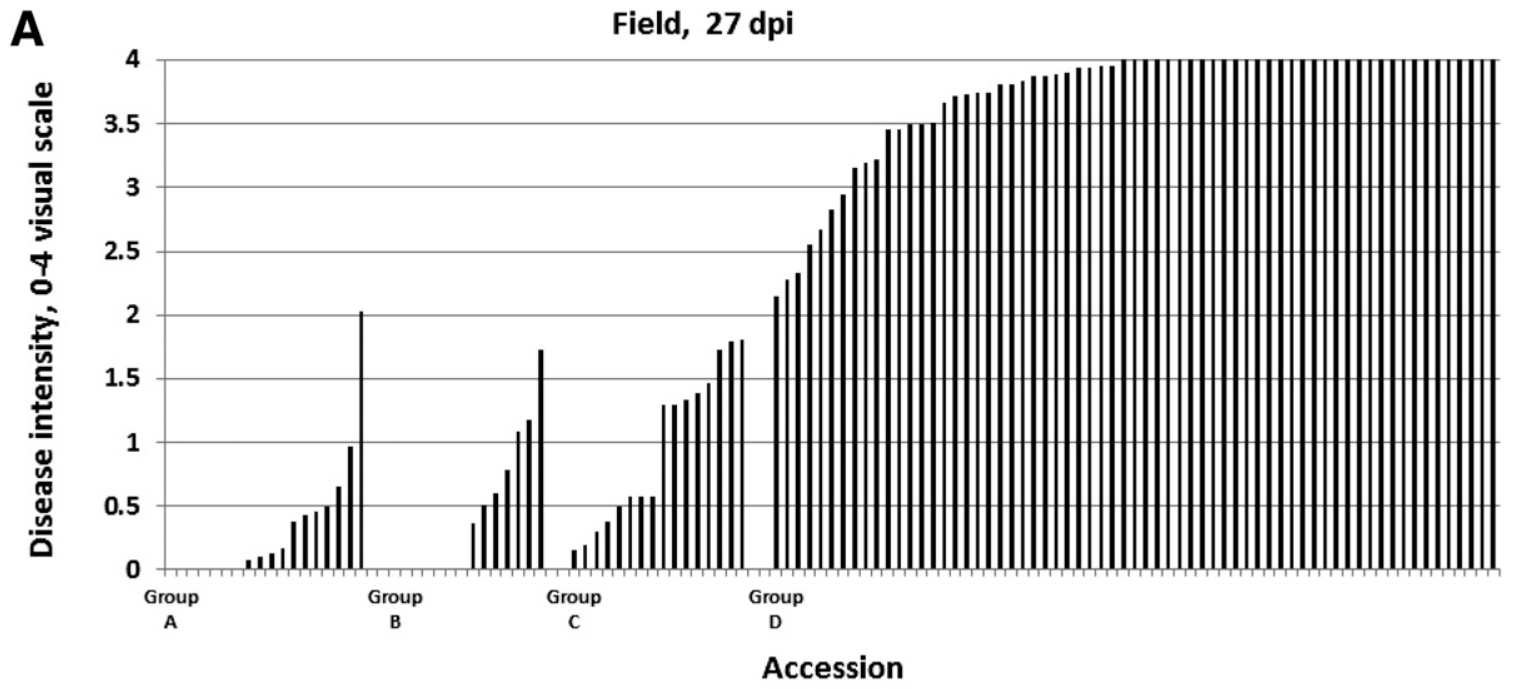

B

Seedlings in growth chambers, $7 \mathrm{dpi}$

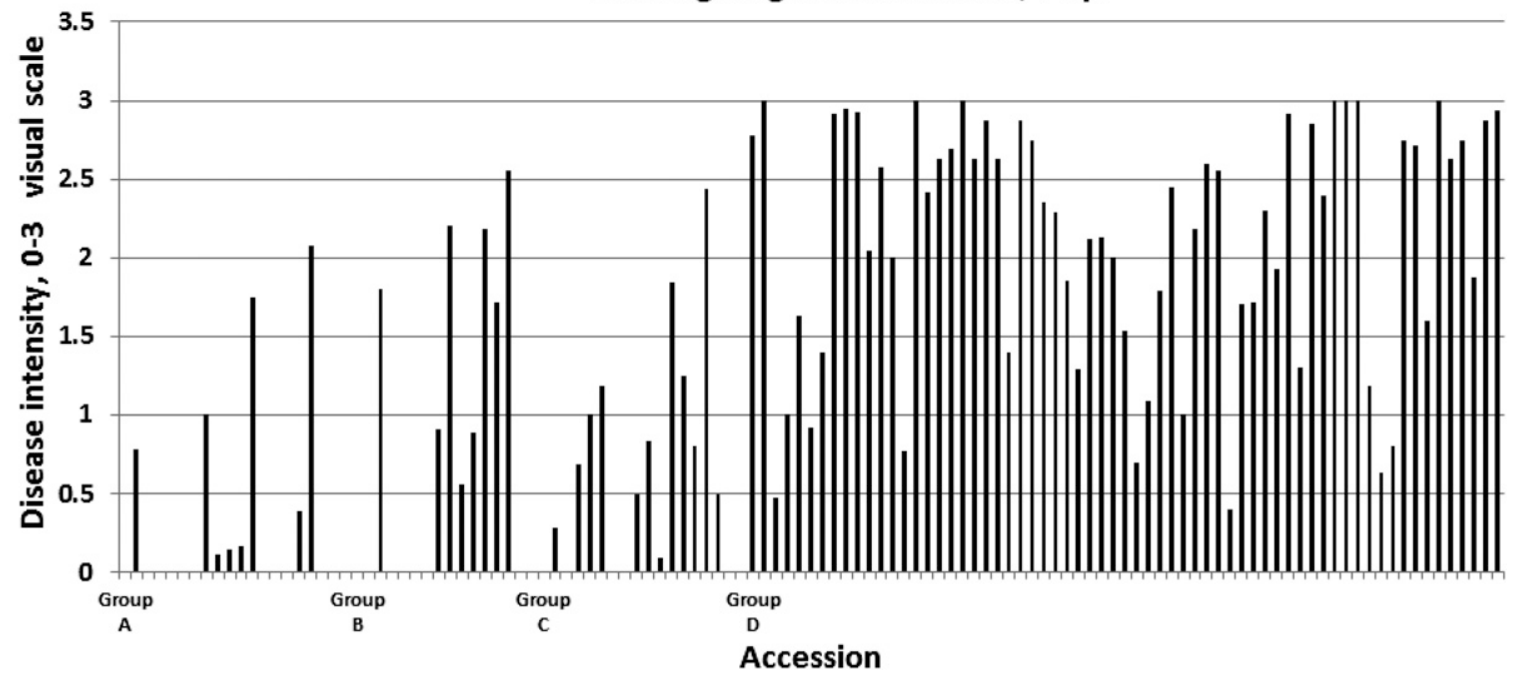

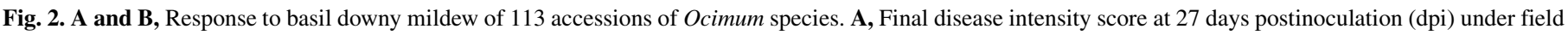

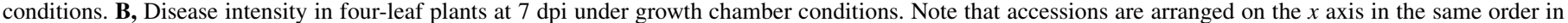
upper and lower images. The list of accessions is shown in Table 1. 
var. citrodorum, $O . \times$ citrodorum, and $O$. basilicum var. minimum). Interestingly, in some susceptible accessions of $O$. basilicum, individual plants were resistant. Thus, among the population of sweet basil (O. basilicum) PI 652070, one single plant showed resistance. After selfpollination, its descendants were resistant in the field. Most commercial $O$. basilicum lines were susceptible to BDM except $O$. basilicum var. citrodorum, $O . \times$ citrodorum, and $O$. basilicum var. minimum, which expressed moderate resistance.
Our results corroborate with Pyne et al. (2014) who showed that PI 652053 (O. americanum var. americanum) was resistant to BDM. PI 172996 and PI 172997 (both O. basilicum var. anisatum), which were resistant to the disease at the young plant stage in their study, were highly resistant in our study at the four-leaf stage and in the field. PI 172998 was reported resistant by Pyne et al. (2014) as well as in our study. The 30 O. basilicum entries reported susceptible by Pyne et al. (2014) were also susceptible in our study.

TABLE 2. Response to downy mildew of 40 Ocimum species in three consecutive seasons under field conditions ${ }^{\mathrm{z}}$

\begin{tabular}{|c|c|c|c|c|c|c|}
\hline \multirow[b]{4}{*}{ Entry } & \multirow[b]{4}{*}{ Accession } & \multirow[b]{4}{*}{ Botanical name } & \multicolumn{4}{|c|}{ Disease intensity } \\
\hline & & & \multirow{2}{*}{ 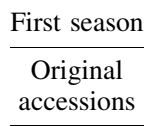 } & \multirow{2}{*}{$\begin{array}{c}\text { Second season } \\
\text { Progeny of a } \\
\text { resistant individual }\end{array}$} & \multicolumn{2}{|c|}{ Third season } \\
\hline & & & & & $\begin{array}{l}\text { Progeny of a } \\
\text { resistant individual }\end{array}$ & $\begin{array}{l}\text { Resistant individual } \times \\
\text { susceptible 'Peri' }(\mathrm{F} 1)\end{array}$ \\
\hline & & & Mean $\pm \mathrm{SD}$ & Mean $\pm S D$ & Mean \pm SD & Mean $\pm \mathrm{SD}$ \\
\hline \multicolumn{7}{|c|}{ Group A } \\
\hline 3 & PI 500945 & O. americanum var. americanum & 0.00 & 0.00 & 0.00 & $0.00^{\mathrm{S}}$ \\
\hline 67 & PI 500942 & O. americanum var. pilosum & 0.00 & & $0.50 \pm 0.00$ & $1.05 \pm 0.37^{\mathrm{S}}$ \\
\hline 69 & PI 500944 & O. americanum var. pilosum & 0.00 & & 0.00 & $0.20 \pm 0.00^{\mathrm{s}}$ \\
\hline 73 & PI 500950 & O. americanum var. pilosum & 0.00 & 0.00 & 0.00 & $0.00^{\mathrm{S}}$ \\
\hline 76 & PI 500954 & O. americanum var. pilosum & 0.00 & 0.00 & $0.25 \pm 0.29$ & $1.05 \pm 0.30^{\mathrm{S}}$ \\
\hline 77 & PI 652052 & O. kilimandscharicum & $0.03 \pm 0.10$ & $0.09 \pm 0.18$ & 0.00 & $1.89 \pm 0.22^{\mathrm{S}}$ \\
\hline 74 & PI 500951 & O. americanum var. pilosum & $0.80 \pm 0.15$ & 0.00 & 0.00 & $0.10 \pm 0.11^{\mathrm{S}}$ \\
\hline 2 & PI 254352 & O. americanum var. americanum & $0.10 \pm 0.17$ & 0.00 & $0.04 \pm 0.09$ & $0.50 \pm 0.00^{\mathrm{S}}$ \\
\hline 72 & PI 500949 & O. americanum var. pilosum & $0.13 \pm 0.23$ & 0.00 & 0.00 & $0.70 \pm 0.00^{\mathrm{s}}$ \\
\hline 54 & PI 652053 & O. americanum var. americanum & $0.17 \pm 0.21$ & 0.00 & 0.00 & \\
\hline 75 & PI 500953 & O. americanum var. pilosum & $0.38 \pm 0.75$ & 0.00 & 0.00 & $1.15 \pm 0.44^{S}$ \\
\hline 1 & PI 253158 & O. americanum var. americanum & $0.43 \pm 0.21$ & $0.30 \pm 0.10$ & $0.02 \pm 0.04$ & $0.46 \pm 0.09^{\mathrm{S}}$ \\
\hline 6 & PI 652062 & O. americanum var. americanum & $0.50 \pm 0$ & $0.50 \pm 0.12$ & & \\
\hline 4 & PI 652058 & O. americanum var. americanum & $0.65 \pm 0.33$ & 0.00 & $0.07 \pm 0.10$ & $1.23 \pm 0.13^{\mathrm{S}}$ \\
\hline 5 & PI 652060 & O. americanum var. americanum & $0.96 \pm 0.37$ & $1.00 \pm 0.30$ & $1.20 \pm 0.18$ & $1.59 \pm 0.14^{\mathrm{S}}$ \\
\hline \multicolumn{7}{|c|}{ Group B } \\
\hline 78 & PI 652066 & O. campechianum & 0.00 & 0.00 & & \\
\hline 79 & PI 652067 & O. gratissimum & 0.00 & 0.00 & & \\
\hline 80 & PI 211715 & O. gratissimum var. gratissimum & 0.00 & 0.00 & & \\
\hline 81 & PI 500952 & O. gratissimum var. gratissimum & 0.00 & 0.00 & & \\
\hline 82 & PI 652055 & O. gratissimum var. macrophyllum & 0.00 & 0.00 & & \\
\hline 64 & PI 414205 & O. tenuiflorum & $0.37 \pm 0.22$ & $1.01 \pm 0.49$ & $0.60 \pm 0.00$ & $1.70 \pm 0.00^{\mathrm{s}}$ \\
\hline 60 & PI 414201 & O. tenuiflorum & $0.60 \pm 0.39$ & $1.11 \pm 0.31$ & $1.50 \pm 0.00$ & $1.50 \pm 0.00^{\mathrm{s}}$ \\
\hline 66 & PI 652059 & O. tenuiflorum & $0.79 \pm 0.41$ & & $0.50 \pm 0.00$ & $1.30 \pm 0.00^{\mathrm{S}}$ \\
\hline 62 & PI 414203 & O. tenuiflorum & $1.09 \pm 0.42$ & $1.35 \pm 0.30$ & $0.50 \pm 0.00$ & $1.40 \pm 0.00^{\mathrm{S}}$ \\
\hline 65 & PI 652056 & O. tenuiflorum & $1.18 \pm 0.27$ & & $0.70 \pm 0.00$ & $1.50 \pm 0.00^{\mathrm{s}}$ \\
\hline \multicolumn{7}{|c|}{ Group C } \\
\hline 107 & Spice basil & Ocimum spp. & $0.15 \pm 0.32$ & $0.64 \pm 0.72$ & & \\
\hline 113 & Blue Spice & Ocimum spp. & $0.30 \pm 0.38$ & $1.02 \pm 0.49$ & & \\
\hline 12 & PI 172998 & O. basilicum var. anisatum & $0.38 \pm 0.18$ & $0.56 \pm 0.15$ & $0.85 \pm 0.14$ & $1.35 \pm 0.09 \mathrm{LF}$ \\
\hline 11 & PI 172997 & O. basilicum var. anisatum & $0.50 \pm 0.09$ & $0.70 \pm 0.21$ & $0.75 \pm 0.20$ & $1.50 \pm 0.00^{\mathrm{LF}}$ \\
\hline 10 & PI 172996 & O. basilicum var. anisatum & $0.57 \pm 0.12$ & $1.13 \pm 0.19$ & $0.90 \pm 0.00$ & $1.32 \pm 0.04^{\mathrm{LF}}$ \\
\hline 94 & Magic Mountain $\mathrm{F} 1^{\mathrm{S}}$ & O. kilimandscharicum $\times O$. basilicum & $0.58 \pm 0.30$ & $1.30 \pm 0.15$ & $1.10 \pm 0.20$ & \\
\hline 95 & Magic White $\mathrm{F} 1^{\mathrm{S}}$ & O. kilimandscharicum $\times$ O. basilicum & $0.58 \pm 0.42$ & $1.25 \pm 0.15$ & $1.20 \pm 0.15$ & \\
\hline 26 & PI 296391 & O. basilicum var. citrodorum & $1.30 \pm 0.77$ & $1.86 \pm 0.24$ & $0.80 \pm 0.00$ & $1.47 \pm 0.08^{\mathrm{F}}$ \\
\hline 86 & Wild Color F1s & O. basilicum & $1.30 \pm 0.21$ & $1.50 \pm 0.29$ & & \\
\hline 8 & PI 170579 & O. basilicum var. minimum & $1.34 \pm 0.31$ & $1.26 \pm 0.23$ & & \\
\hline 14 & PI 174284 & O. basilicum var. anisatum & $1.38 \pm 1.16$ & $0.92 \pm 0.21$ & $0.80 \pm 0.00$ & $1.56 \pm 0.22^{\mathrm{LF}}$ \\
\hline 55 & PI 652054 & O. basilicum var. citrodorum 'Mrs. Burns' & $1.47 \pm 0.31$ & $1.64 \pm 0.20$ & $1.50 \pm 0.00$ & $1.50 \pm 0.00^{\mathrm{S}}$ \\
\hline 23 & PI 253157 & O. basilicum var. citrodorum & $1.73 \pm 1.04$ & $1.11 \pm 0.28$ & $1.12 \pm 0.26$ & $1.41 \pm 0.32^{\mathrm{F}}$ \\
\hline 105 & Lemon basil & O. basilicum var. citrodorum & $1.79 \pm 0.54$ & $2.03 \pm 0.54$ & & \\
\hline 84 & Ajaka F1 ${ }^{\mathrm{S}}$ & O. basilicum & $1.81 \pm 0.26$ & $1.70 \pm 0.21$ & & \\
\hline \multicolumn{7}{|c|}{ Group D } \\
\hline 102 & Sweet Danni & O. $\times$ citrodorum & $2.15 \pm 0.25$ & $2.32 \pm 0.25$ & & \\
\hline 37 & PI 368697 & O. basilicum & $2.28 \pm 1.24$ & $1.21 \pm 0.24$ & $0.99 \pm 0.21$ & \\
\hline 13 & PI 173746 & O. basilicum & $2.95 \pm 0.93$ & $1.93 \pm 0.34$ & $1.50 \pm 0.13$ & $1.68 \pm 0.29^{\mathrm{F}}$ \\
\hline 109 & Rubin & O. basilicum & $3.15 \pm 0.32$ & $3.77 \pm 0.63$ & & \\
\hline 58 & PI 652070 & O. basilicum & $3.46 \pm 1.21$ & $0.24 \pm 0.46$ & $0.92 \pm 0.30$ & $1.42 \pm 0.20^{\mathrm{F}}$ \\
\hline 112 & Gecofure basil & O. basilicum 'Genovese' & $3.73 \pm 0.34$ & $4.00 \pm 0.00$ & & \\
\hline 100 & Peri & O. basilicum Sweet basil 'Peri' & $3.95 \pm 0.16$ & $3.61 \pm 0.53$ & $3.08 \pm 0.71$ & \\
\hline 101 & Genoveser 142 & O. basilicum 'Genovese' & $4.00 \pm 0.00$ & $4.00 \pm 0.00$ & & \\
\hline
\end{tabular}

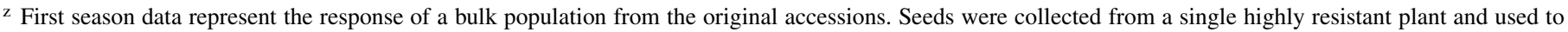
test the response to basil downy mildew (BDM) at 27 days postinoculation (dpi) in a second and a third season. During the second season a cross was made between one resistant individual and the susceptible sweet basil Peri. The response to BDM of the F1 plants was recorded at 39 dpi. Final score is represented by mean \pm standard deviation of the mean (SD). The uppercase letters represent sterility (S), fertility (F), or low fertility (LF) of the F1 hybrids. 


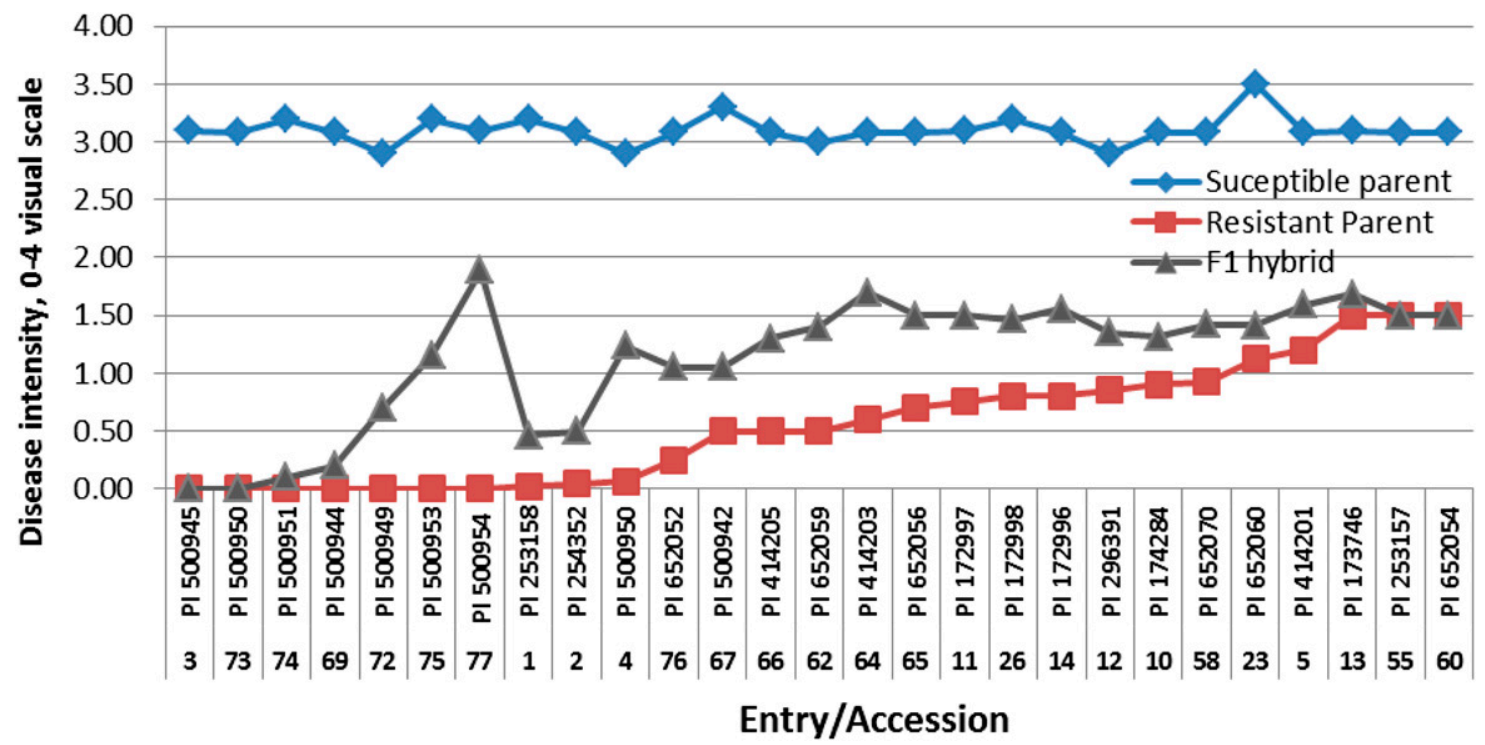

Fig. 3. Response to basil downy mildew of 27 F1 hybrids of Ocimum species made by crossing resistant parental accessions with the susceptible cultivar Peri.

Wyenandt et al. (2010) evaluated the response to BDM of 30 basil entries in field trials in southern and northern New Jersey. The cultivars Spice, Blue Spice, and Blue Spice F1 showed neither visible symptoms nor sporulation, suggesting potential sources of resistance in Ocimum spp., while three $O$. citrodorum entries showed symptoms with light sporulation. In our study, Spice and Blue Spice usually showed some symptoms but no sporulation (except in the second season, Table 2), while O. citrodorum entries (group C) responded similarly showing symptoms with light sporulation.

The crosses we made between the highly resistant (or resistant) parents and the susceptible parent Peri provided information on the versatility of the resistance genes in the collection we have studied (Fig. 3). These data suggest that three genotypes (PI 500945, PI 500950, and PI 500951) carried dominant gene(s) for resistance while others probably carried partially dominant genes. Interestingly, one resistant genotype probably carried a recessive gene for resistance. This variety of genes may be used in breeding programs to ensure durable resistance against BDM.

The F1 offspring plants obtained from the crosses between the resistant $O$. americanum entries and the susceptible $O$. basilicum 'Peri' were infertile, and therefore could produce no F2 progeny plants. This genetic barrier, derived mainly from differential ploidy of the two species, prevents further exploitation of these important resistance genes. Current studies should be directed to overcome this genetic barrier by embryo rescue and genome duplication techniques.

\section{LITERATURE CITED}

Aghaei, M., Darvishzadeh, R., and Hassani, A. 2012. Molecular characterization and similarity relationships among Iranian basil (Ocimum basilicum L.) accessions using inter simple sequence repeat markers. Rev. Cienc. Agronom. 43:312-320

Belbahri, L., Calmin, G., Lefort, F., and Pawlowski, J. 2005. Phylogenetic analysis and real-time PCR detection of a new Peronospora species responsible for downy mildew disease of sweet basil and sage. Mycol. Res. 109:1276-1287.

Carovic-Stanko, K., Liber, Z., Besendorfer, V., Javornik, B., Bohanec, B., Kolak, I., and Satovic, Z. 2010. Genetic relations among basil taxa (Ocimит L.) based on molecular markers, nuclear DNA content, and chromosome number. Plant Syst. Evol. 285:13-22.

Cohen, Y., Vaknin, M., Ben-Naim, Y., and Rubin, A. E. 2013a. Light suppresses sporulation and epidemics of Peronospora belbahrii. PLoS One 8: e81282.
Cohen, Y., Vaknin, M., Ben-Naim, Y., Rubin, A. E., Galperin, M., Silverman, D., Bitton, S., and Adler, U. 2013b. First Report of the occurrence and resistance to mefenoxam of Peronospora belbahrii, causal agent of downy mildew of basil (Ocimum basilicum) in Israel. Plant Dis. 97:692.

Elgayyar, M., Draughon, F. A., Golden, D. A., and Mount, J. R. 2001. Antimicrobial activity of essential oils from plants against selected pathogenic and saprophytic microorganisms. J. Food Prot. 64:1019-1024.

Farahani-Kofoet, R. D., Roemer, P., and Grosch, R. 2012. Systemic spread of downy mildew in basil plants and detection of the pathogen in seed and plant samples. Mycol. Prog. 11:961-966.

Farahani-Kofoet, R. D., Römer, P., and Grosch, R. 2014. Selecting basil genotypes with resistance against downy mildew. Sci. Hortic. (Amsterdam) 179:248-255.

Garibaldi, A., Bertetti, D., and Gullino, M. L. 2007. Effect of leaf wetness duration and temperature on infection of downy mildew (Peronospora sp.) of basil. J. Plant Dis. Prot. 114:6-8.

Gilardi, G., Demarchi, S., Garibaldi, A., and Gullino, M. L. 2013. Management of downy mildew of sweet basil (Ocimum basilicum) caused by Peronospora belbahrii by means of resistance inducers, fungicides, biocontrol agents and natural products. Phytoparasitica 41:59-72.

Garibaldi, A., Minuto, A., and Gullino, M. L. 2005. First report of downy mildew caused by Peronospora sp. on basil (Ocimum basilicum) in France. Plant Dis. 89:683.

Garibaldi, A., Minuto, A., Minuto, G., and Gullino, M. L. 2004a. First report of downy mildew on basil (Ocimum basilicum) in Italy. Plant Dis. 88:312-312.

Garibaldi, A., Minuto, G., Bertetti, D., and Gullino, M. L. 2004b. Seed transmission of Peronospora sp. of basil. J. Plant Dis. Prot. 111:465-469.

Hansford, C. G. 1933. Annual report of the mycologist. Rev. Appl. Mycol. 12: 421-422.

Harley, R. M., and Heywood, C. A. 1992. Chromosome numbers in tropical American Labiatae. Advances in Labiatae Science. R. M. Harley and T. Reynolds, eds. Royal Bot. Garden, London.

Homa, K., Barney, W. P., Ward, D. L., Wyenandt, C. A., and Simon, J. E. 2014. Evaluation of fungicides for the control of Peronospora belbahrii on sweet basil in New Jersey. Plant Dis. 98:1561-1566.

Hussain, A. I., Anwar, F., Hussain Sherazi, S. T., and Przybylski, R. 2008. Chemical composition, antioxidant and antimicrobial activities of basil (Ocimum basilicum) essential oils depends on seasonal variations. Food Chem. 108:986-995.

Koroch, A. R., Wang, W., Michael, T. P., Dudai, N., Simon, J. E., and Belanger, F. C. 2010. Estimation of nuclear DNA content of cultivated Ocimum species by using flow cytometry. Isr. J. Plant Sci. 58:183-189.

McLeod, A., Coertze, S., and Mostert, L. 2006. First report of a Peronospora species on sweet basil in South Africa. Plant Dis. 90:1115.

Paton, A., and Putievsky, E. 1996. Taxonomic problems and cytotaxonomic relationships between and within varieties of Ocimum basilicum and related species (Labiatae). Kew Bull. 51:509-524.

Paton, A., Harley, M. R., and Harley, M. M. 1999. Ocimum: An overview of classification and relationships. Basil the Genus Ocimum. R. Hiltunen and Y. Holm, eds. Harwood Academic Publishers, Amsterdam, The Netherlands.

Phippen, W. B., and Simon, J. E. 2000. Anthocyanin inheritance and instability in purple basil (Ocimum basilicum L.). J. Hered. 91:289-296. 
Pyne, R. M., Koroch, A. R., Wyenandt, C. A., and Simon, J. E. 2014. A rapid screening approach to identify resistance to basil downy mildew (Peronospora belbahrii). HortScience 49:1041-1045.

Roberts, P. D., Raid, R. N., Harmon, P. F., Jordan, S. A., and Palmateer, A. J. 2009. First report of downy mildew caused by a Peronospora sp. on basil in Florida and the United States. Plant Dis. 93:199.

Ronco, L., Rollán, C., Choi, Y. J., and Shin, H. D. 2009. Downy mildew of sweet basil (Ocimum basilicum) caused by Peronospora sp. in Argentina. Plant Pathol. 58:395.

Simon, J. E., Quinn, J., and Murray, R. G. 1990. Basil-A source of essential oils. Advances in New Crops Research. J. Janick and J. E. Simon, eds. Timber Press, Portland, OR.
Suppakul, P., Miltz, J., Sonneveld, K., and Bigger, S. W. 2003. Antimicrobial properties of basil and its possible application in food packaging. J. Agric. Food Chem. 51:3197-3207.

Thines, M., Telle, S., Ploch, S., and Runge, F. 2009. Identity of the downy mildew pathogens of basil, coleus, and sage with implications for quarantine measures. Mycol. Res. 113:532-540.

Vieira, R. F., and Simon, J. E. 2006. Chemical characterization of basil (Ocimum spp.) based on volatile oils. Flavour Fragrance J. 21:214-221.

Wyenandt, C. A., Simon, J. E., McGrath, M. T., and Ward, D. L. 2010. Susceptibility of basil cultivars and breeding lines to downy mildew (Peronospora belbahrii). HortScience 45:1416-1419. 